\title{
CASE REPORT OF IDIOPATHIC BILATERAL ORBITAL MYOSITIS
}

\author{
Fatma Şimşek ${ }^{1}$ \\ Correspondence: klamaks@hotmail.com \\ ${ }^{1}$ Ataturk Univercity Faculty of Medicine, Neurology, Erzurum, Turkey
}

\author{
Article History: \\ Received: September 23, 2020 \\ Accepted: April 21, 2021 \\ Published: July 1, 2021 \\ Cite this as: \\ Şimşek F. Case report of \\ idiopathic bilateral orbital \\ myositis. Malang Neurology \\ Journal; 2021.7: 134-136. \\ http://dx.doi.org/10.21776/ub.mnj \\ .2021.007.02.10
}

Keywords: Bilateral involvement, magnetic resonance imaging, steroids

\begin{abstract}
Orbital myositis is an inflammatory disease affecting extraocular muscles. Mostly unilateral and rarely bilateral orbital involvement is seen. Bilateral involvement is a secondary table to systemic diseases and recurrence can be seen. Diagnosis is made by clinical, examination and imaging. Increased density of extraocular muscles and increased muscle mass should be seen in computed tomography or magnetic resonance imaging. The basis of the diagnosis is exclusion and the differential diagnosis needs to be done well. Steroids and other immunosuppressive agents may be used in the treatment. Patients respond dramatically to steroid treatment. While short-term steroid treatment may be sufficient in idiopathic patients, orbital myositis secondary to systemic disease requires longer-term and non-steroid immunosuppressive treatments. Here, a case of idiopathic orbital myositis with bilateral involvement is presented as it is a rare condition.
\end{abstract}

ABSTRACT

\section{Introduction}

Orbitalmiositis (OM) is an idiopathic, non-infectious, inflammatory disease involving extraocular muscles, characterized by acute onset orbital pain accompanied by diplopia, ptosis, chemosis and conjunctival injection. ${ }^{1,2}$ Women are affected more in a 2:1 ratio. ${ }^{3,4}$ OM may be unilateral or bilateral. In the series of 75 cases of Siatkowski et al., the rate of cases with a single muscle involvement was observed as $68 \%$, the involvement of two muscles was $22 \%$, the involvement of three or more muscles was $10 \%$, and bilateral involvement was observed in only one case. ${ }^{4}$ Usually muscle tendon involvement and fusiform enlargement of the muscle body are monitored in Computed tomography (CT) or magnetic resonance imaging (MRI). The diagnosis is made clinically and radiologically. It may be idiopathic as well as there are many reasons accused in the etiology such as infectious causes, autoimmune diseases, paraneoplastic, systemic inflammatory diseases and drug reactions. We present this case because idiopathic bilateral OM is a rare condition.

\section{Case Report}

The 52-year-old female patient presented to our neurology outpatient clinic with complaints of conjunctival redness, burning, orbital pain that started 10 days before in the right eye and 2-3 days later in the left eye. She described occasional double vision and had no vision loss. Anamnesis: she had bipolar mood disorder and was using valproic acid $1000 \mathrm{mg} /$ day and quetiapine $100 \mathrm{mg} /$ day for two years.. In physical examination; she had conjunctival redness in both eyes, edema of the eyelids (Figure 1), no exophthalmos was present. She had no fever and vital signs were stable. Visual acuity was 20/20 in both eyes and fundus was normal. In neurological examination; upward, inward and outward sight was restricted in right eye, inward and outward sight was limited in left eye. There was no ptosis, and the light reflex was normal in bilateral direct and indirect. There was no weakness in the musculus orbicularis oris force. Other neurological examination was normal. Complete blood count, erythrocyte sedimentation rate, C-reactive protein, basic metabolites, anti-nuclear antibody were normal. Anti TSHR:0,334 U/L (<1,58 U/L negative), TSH: $3,53 \mu \mathrm{IU} / \mathrm{mL}$, FT4: $0,68 \mu \mathrm{IU} / \mathrm{mL}$ and normal. Orbital and brain MRI revealed increased densities in left eye medial rectus, right eye medial and lateral rectus, and enlargement of muscle mass (Figure 2). No pathology was observed in the cavernous sinus structures. MRI angiography requested for carotid-cavernous fistula was normal. The patient was hospitalized with the diagnosis of $\mathrm{OM}$ and steroid treatment was started from $1 \mathrm{mg} / \mathrm{kg}$. It was seen that the patient showed dramatic improvement after 3 days. In control orbital CT performed 3 weeks later, enlargement of the muscle mass in the superior rectus of the only right eye was observed (Figure 3). Clinically, the patient recovered almost completely, with mild redness of the eyes, no edema, and with free eye movements (Figure 4). Steroid treatment was continued from $1 \mathrm{mg} / \mathrm{kg}$ for one month. At the end of one month, the steroid dose was gradually reduced to $10 \mathrm{mg} /$ day and recommended to continue for 5-6 months. No complication or recurrence was observed in patient after the treatment. Written informed consent was obtained from the patient for use of her pictures. 

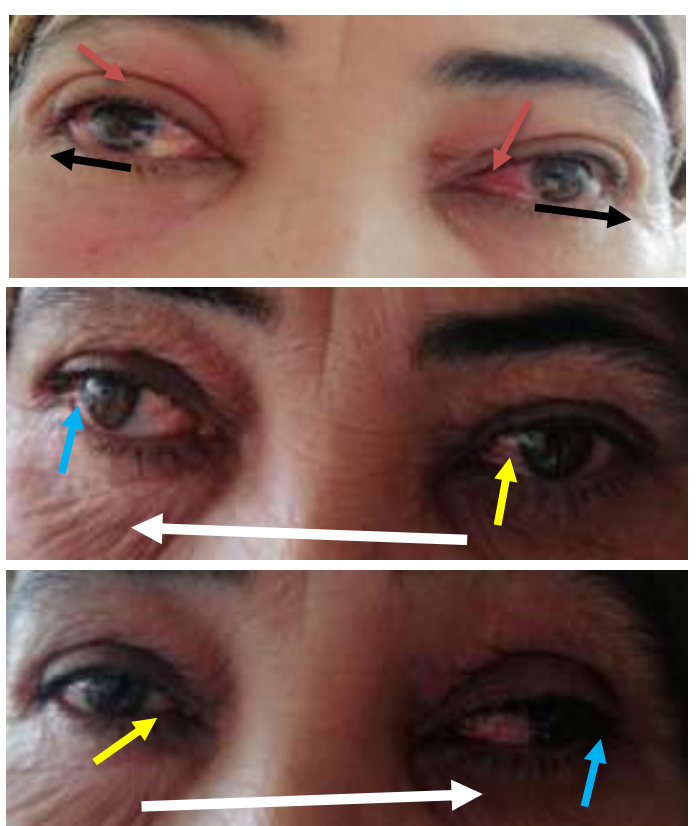

Figure 1. Conjunctival redness, edema (red arrow), outward deviation of the eyes (black arrow), bilateral inward (yellow arrow) and outward (blue arrow) sight of the patient were seem to be limited in both eyes. (white arrows show the direction of view)

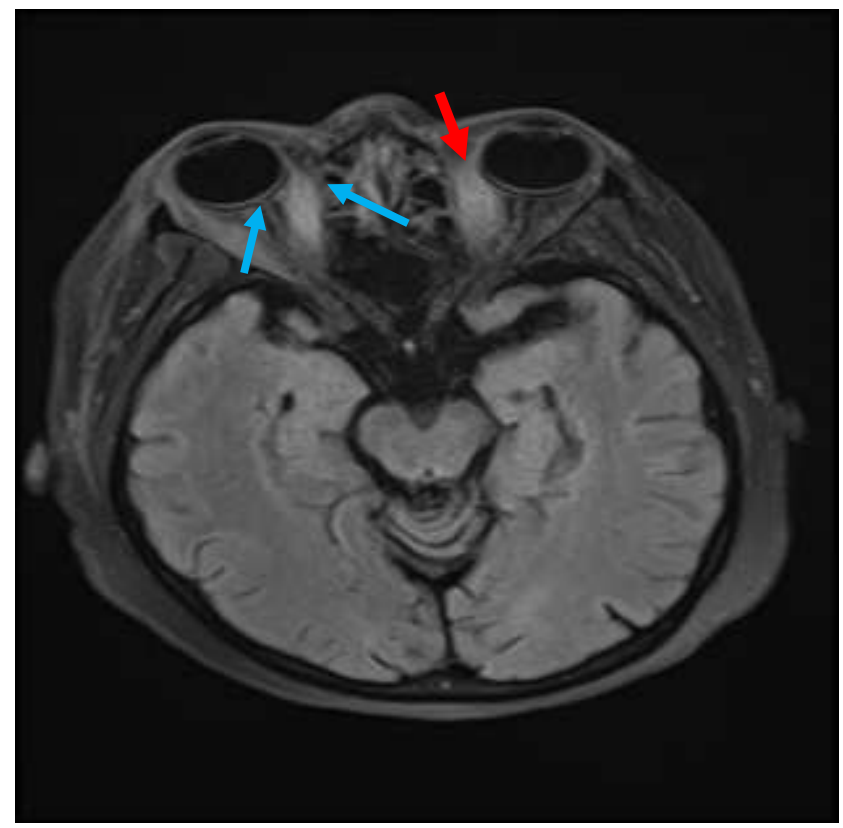

Figure 2. T2 flair MRI reveals an increase in density and an increase in muscle mass in the medial rectus of the left eye (red arrow), medial and lateral rectus of the right eye (blue arrow)

\section{Discussion}

$\mathrm{OM}$ is a well-established inflammatory disorder and requires careful examination for differential diagnosis. The diagnosis is made by a good anamnesis, clinical, examination findings and imaging (CT or MRI).

Differential diagnosis of idiopathic OM includes infections (viral infections, orbital cellulitis, orbital abscess, Lyme disease, Herpes zoster and syphilis), inflammatory reaction (trauma, foreign body, bisphosphonate-related reaction and postvaxinal reaction), Tolosa - Hunt syndrome, thyroid ophthalmopathy, vasculitis (Wegener's granulomatosis, polyarteritis) nodosa, rheumatoid arthritis, giant cell arteritis and Kawasaki disease), systemic lupus erythematosus, sarcoidosis, inflammatory bowel disease,

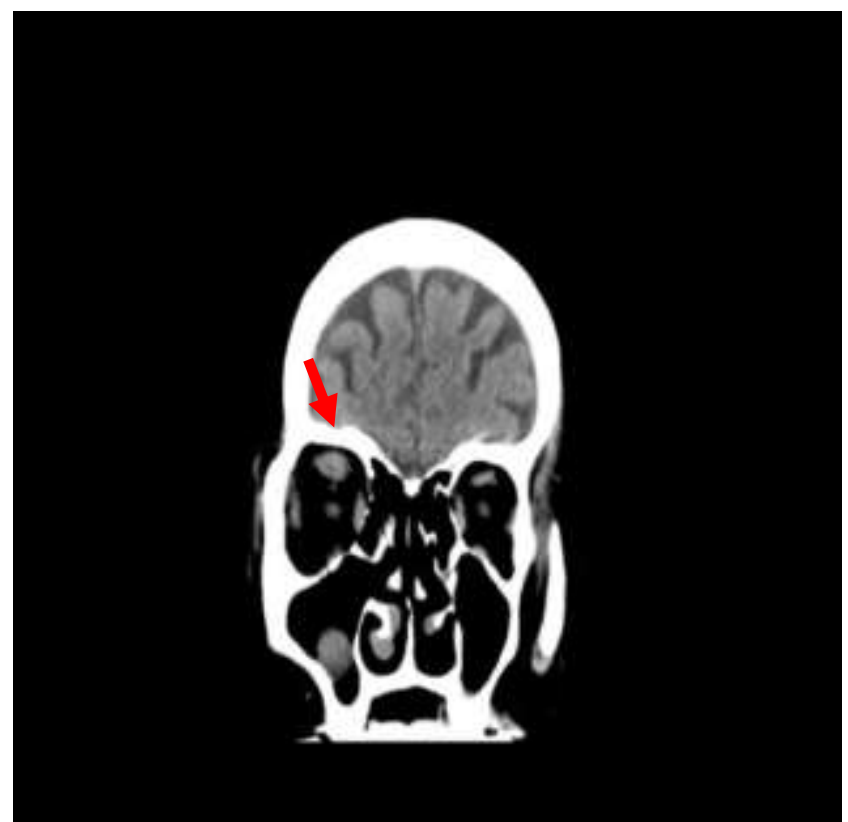

Figure 3. Orbital CT reveals enlargement of muscle mass in the superior rectus of the right eye (red arrow). It is observed that the increase in muscle mass seen in the medial and lateral rectus before the treatment resolves after the treatment.

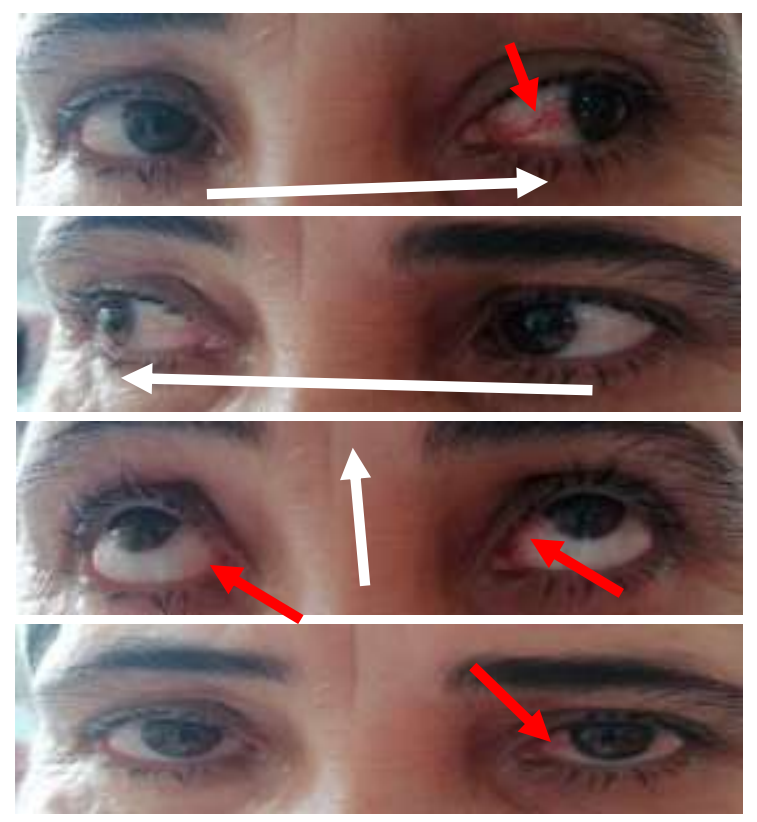

Figure 4. Eye movements appear to be free (white arrows show the direction of view)and mild conjunctival hyperemia (red arrow) is observed two weeks after medication.

neoplasia, arteriovenous fistula and malformations. ${ }^{5}$ The diagnosis is based on exclusion in idiopathic OM. Therefore, all reasons that may lead to the present clinical picture should be excluded. The most commonly involved muscles in patients are medial and lateral rectus, bilateral involvement rate is low and predominantly seen in female gender. ${ }^{2}$ Complete blood count, erythrocyte sedimentation rate, C-reactive protein, liver and kidney function tests, 
thyroid function tests, anti-nuclear antibody and antibodies specific for connective tissue diseases should be requested in all patients with suspected idiopathic OM. These assays performed for our patient were normal. Observing increased densities in left eye medial rectus, right eye medial and lateral rectus, and enlargement of muscle mass in cranial and orbital MRI requested for our patient prediagnosed with OM supported the diagnosis. The patient had bilateral conjunctival hyperemia in the eyes at the first admission and no inflammatory event was considered by the eye clinic. MRI angiography requested for suspicion of caroticocavernous fistula due to the present appearance was normal. No pathology was observed in the cavernous sinus on brain MRI.

Bilateral involvement in our patient suggested thyroid ophthalmopathy in the foreground. Thyroid ophthalmopathy is one of the diseases in the differential diagnosis of OM. While $\mathrm{OM}$ is usually unilateral and mostly involved in medial rectus muscle, involvement is usually bilateral in thyroid ophthalmopathy. In thyroid ophthalmopathy, propitosis is more prominent and diplopia is rare. Orbital pain is more prominent in OM. Visual loss may be seen in low rate in thyroid ophthalmopathy, but not expected in OM. Antibody positivity and systemic symptoms are observed in thyroid ophthalmopathy. Our patient had no known thyroid disease, and antibodies and TFT were normal. Orbital pain and diplopia were prominent and there was no decrease in visual acuity. Our patient was evaluated as OM by clinical, laboratory and imaging studies.

Multiple muscle involvement in OM and being affected in both eyes are seen as secondary to a systemic disease (such as sarcoidosis, systemic lupus erythematosus, rheumatoid arthritis, scleroderma) and recurrence rate is higher. In the literature, there are cases with bilateral OM presenting with active bowel symptoms and diagnosed as ulcerative colitis. ${ }^{6}$ Our patient had no known history of systemic disease except for systemic complaints and bipolar disorder. No recurrence was seen in one-year follow-up. With the present table, our patient was accepted as idiopathic OM.

In conclusion, may be seen as bilateral, even rare. Bilateral involvement is mostly associated with systemic diseases, but it may rarely be idiopathic as in our patient. While short-term steroid therapy may be sufficient in idiopathic patients, additional immunosuppressive therapies are needed in patients with underlying systemic disease.

\section{Conclusion}

Bilateral involvement is mostly associated with systemic diseases, but it may rarely be idiopathic as in our patient. While short-term steroid therapy may be sufficient in idiopathic patients, additional immunosuppressive therapies are needed in patients with underlying systemic disease.

\section{Acknowledgement}

None.

\section{Conflict of Interest}

Authors declare that we have no conflict of interest.

\section{References}

1. Montagnese F, Wenninger S, Schoser B. Orbiting around the orbital myositis: Clinical features, differential diagnosis and therapy. Journal of Neurology; 2016. 263(4):631-40.

DOI: $10.1007 / \mathrm{s} 00415-015-7926-\mathrm{X}$

2. Schoser BG. Ocular myositis: Diagnostic assessment, differential diagnoses, and therapy of a rare muscle disease-five new cases and review. Clinical Ophthalmology (Auckland, NZ); 2007. 1(1):37. Avalaible form:

https://www.ncbi.nlm.nih.gov/pmc/articles/PMC2699 981/

3. Scott IU, Siatkowski RM. Idiopathic orbital myositis. Current Opinion in Rheumatology; 1997. 9(6):504-12. DOI: 10.197/00002281-199711000-00005

4. Siatkowski RM, Capo H, Byrne SF, Gendron EK, Flynn JT, Munoz M, et al. Clinical and echographic findings in idiopathic orbital myositis. American Journal of Ophthalmology; 1994. 118(3):343-50. DOI: 10.1016/S0002-9394(14)72959-9

5. Benmiloud S, Boubbou M, Hida M. Bilateral idiopathic orbital myositis in an infant. Int J Res Med; 2013.2:112-4. Avalaible from: https://ijorim.com/siteadmin/article_issue/1389900573 28_Sarra\%20Benmiloud.pd

6. Bennion J, Harris MA, Sivak-Callcott JA, Nguyen J. Bilateral diffuse orbital myositis in a patient with relapsing ulcerative colitis. Ophthalmic Plastic \& Reconstructive Surgery; 2012. 28(5):e119-e20. DOI: $10.1097 /$ IOP.0b013e318244a34e 